\title{
Milli Pompalarda Dalma Derinliği Değişiminin Bazı Pompaj Parametrelerine Etkileri
}

\section{Nuri ORHAN ${ }^{1}$, Ali Yavuz SEFLEK ${ }^{2}$, Osman ÖZBEK ${ }^{3}$}

Selçuk Üniversitesi Ziraat Fakültesi Tarım Makineleri ve Teknolojileri Mühendisliği Bölümü

${ }^{1}$ https://orcid.org/0000-0002-9987-1695, ${ }^{2}$ https://orcid.org/0000-0003-1009-6635, ${ }^{3 h t t p s: / / o r c i d . o r g / 0000-0003-0034-9387 ~}$

凶: nuriorhan@selcuk.edu.tr

\section{ÖZET}

$\mathrm{Bu}$ çalışmada milli pompaların farklı dalma derinliklerinin pompaj parametrelerine etkilerinin incelenmesi amaçlanmıştır. Farklı anma çapına sahip üç adet milli pompanın $\left(\mathrm{M}_{1}, \mathrm{M}_{2}, \mathrm{M}_{3}\right)$ üç farklı su giriş alanlarında $\left(\mathrm{KA}_{1}, \mathrm{KA}_{2}, \mathrm{KA}_{3}\right)$ ve farkl dalma derinliklerinde (S) pompaların debi $(\mathrm{Q})$, çıkış basıncı $(\mathrm{Pb})$, şebekeden çekilen güç $(\mathrm{N})$ ve gürültü seviyeleri (G) ölçülmüş, toplam dinamik yükseklik (TDY) değeri ise hesaplanmıştır. Her üç milli pompa anma çapı ve su giriş kesit alanı seviyelerinde debinin artması ile TDY değerleri azalmıştır. $\mathrm{M}_{1}$ (Ø $78 \mathrm{~mm}$ ), $\mathrm{M}_{2}$ (Ø 105mm) ve $\mathrm{M}_{3}$ (Ø $128 \mathrm{~mm}$ ) pompalarının tüm debi, su giriş kesit alanları ve farklı dalma derinliklerinde şebekeden çektikleri güç ortalamaları sırasıyla 5.06-5.2-7.21 kW, gürültü seviyeleri ortalamaları ise sirasıyla 78.58-80.06 ve 81.2 dBA olarak ölçülmüştür. Pompa anma çaplarının artması hem şebekeden çekilen güç değerlerini hem de gürültü seviyelerini artırmıştır. Pompaların şebekeden çektikleri güç bakımından $\% 20$ büyütülmüş su giriş kesitinin, gürültü seviyesi bakımından ise orijinal su giriş kesitinin kullanılmasının uygun olduğu ortaya çıkmıştır. Dalma derinliklerinin çok düşürülmesi sonucu vorteks oluşmuş ve pompalara hava girişi olmuştur. Bu durumun sonucunda TDY ve şebekeden çekilen güç değerlerinde ani azalmalar, gürültü seviyelerinde ani yükselmeler ölçülmüştür. Pompaların TDY, çektikleri güç ve gürültü seviyeleri değişimlerinin düzenli takibi ile verimli çalışmaları sağlanabilir.
Araştırma Makalesi

Makale Tarihçesi

Geliş Tarihi : 04.05.2020

Kabul Tarihi : 03.12.2020

Anahtar Kelimeler

Dalma Derinliği

Gürültü

Vorteks

Milli Pompalar

\section{Effects of Submergence Change in Shaft Pumps on Some Pump Parameters}

\section{ABSTRACT}

The aim of this study was to investigate the effects of different plunging depths of the shaft pumps on the pumping parameters. In three different nominal diameters of pumps $\left(\mathrm{M}_{1}, \mathrm{M}_{2}, \mathrm{M}_{3}\right)$ three different water inlet areas $\left(\mathrm{KA}_{1}, \mathrm{KA}_{2}, \mathrm{KA}_{3}\right)$ and in different submergence $(\mathrm{S})$ output pressure $(\mathrm{Pb})$, power and noise levels were examined, the pumps total dynamic head (TDH) were calculated. TDH values decreased with the increasing flowrate of the pumps in each nominal diameter and water inlet cross-section. $\mathrm{M}_{1}(\varnothing 78 \mathrm{~mm})$, $\mathrm{M}_{2}$ (Ø $105 \mathrm{~mm}$ ) and $\mathrm{M}_{3}$ (Ø $128 \mathrm{~mm}$ ) pumps average power drawn from the mains in all flow, water inlet cross-section and different submergence were measured as $5.06-5.2-7.21 \mathrm{~kW}$ and noise levels averaged as $78.58-80.06$ and $81.2 \mathrm{dBA}$, respectively. Increasing the pump nominal diameters increased both the power values drawn from the network and the noise levels. In the pumps, it has been found that it was appropriate to use the water inlet section $20 \%$ enlarged in terms of the power drawn from the mains, and the water inlet section orginal in terms of the noise level. As a result of the very low submergence, vortexes have formed and air enters the pumps. As a result of this situation, a sudden decrease in power drawn from the mains and TDY values, a sudden increase in noise levels were measured. Efficient operation can be achieved by measuring regularly the changes in power drawn from the mains, TDY and noise levels of the pumps.

\section{Research Article}

$\begin{array}{ll}\text { Article History } & \\ \text { Received } & : 04.05 .2020 \\ \text { Accepted } & : 03.12 .2020\end{array}$

Keywords

Submergence

Noise

Vortex

Shaft Pumps 


\begin{tabular}{ll}
\hline Atıf İçin: & Orhan N, Şeflek AY, Özbek O, 2021 Milli Pompalarda Dalma Derinliği Değişiminin Bazı Pompaj \\
& Parametrelerine Etkisi. KSÜ Tarım ve Doğa Derg 24 (4): 747-756. DOI: 10.18016/ksutarimdoga.vi.732012. \\
To Cite: & Orhan N, Şeflek AY, Özbek O, 2021. Effects of Submergence Change in Shaft Pumps on Some Pump Parameters. \\
& KSU J. Agric Nat 24 (4): 747-756. DOI: 10.18016/ksutarimdoga.vi.732012. \\
\hline
\end{tabular}

\section{Gíniş}

Tarımsal açıdan büyük öneme sahip olan su, akarsulardan, göllerden, göletlerden vb. kaynaklardan yeterince ve uygun şartlarda elde edilemeyince yeraltı su kaynakları devreye girmektedir. Tarıma açılan arazi varlığımızın yaklaşık \%20'sini oluşturan sulamaya açılmış arazinin \%38'i kuyulardan, \%28'i akarsulardan, \%10'u kaynaklardan, \%3’ü göletlerden geriye kalan kısımlar ise diğer su kaynaklarından sağlanmaktadır (Anonim 2016). Görüldüğü gibi sulanan arazilerimizin büyük çoğunluğunda yer altı su kaynaklarımız kullanılmaktadır. Yer altından suyu yüzeye çıkarmak için derin kuyu pompaları kullanılmaktadır. Bir kuyunun derin kuyu olarak isimlendirilebilmesi için, kuyu derinliğinin yüzeyden en az $7 \mathrm{~m}$ derinlikte olması gerekmektedir. Derin kuyu pompaları özel olarak açılan derin kuyulardan su sağlamak için kullanılır. Derin kuyu pompaları kademeli olarak yapılır ve kademe sayısına bağlı olarak 300-400 m derinlikteki suyu yer yüzeyine çıkartabilirler. Sondaj makinaları ile açılan bu kuyulara uygun çap ve kademe sayısına sahip derin kuyu pompaları belirli standartlar altında yerleştirilir. Kuyuya yerleştirilen derin kuyu pompalarının debisi genel olarak açılan kuyunun debisi ile sınırlıdır. Pompa debisi ile kuyu debisi birbirine uyumlu olmalıdır. Kuyu debisinin düşük olması halinde su seviyesi düşer ve pompa emişini kaybeder. Bu sebeple kuyu ve pompa benzer karakteristikleri içermelidir (Çalışır 2009; Driscoll 2010).

Pompa su giriş ağzı ile su seviyesi arasındaki düşey mesafe dalma derinliği olarak adlandırılmaktadır. Pompaj esnasında dalma derinliğindeki aşırı azalmalar (su seviyesindeki aşırı düşümler) pompanın su giriş ağzında vorteks oluşmasına hatta pompa içerisine hava girmesine sebep olur (Chen ve ark. 2012). Pompa içerisine giren hava kavitasyon oluşmasına sebep olabilir. Kavitasyon pompaların ekonomik ömrünü ve verimlerini olumsuz yönde etkiler (Nagahara ve ark. 2001; Yıldırım ve ark. 2011).

Kuyulardaki su seviyelerinin azalmasına etkili diğer etkenler iklim değişikliği ve bilinçsiz/aşırı sulamalardır. Göçmez ve ark. (2008) Konya'da yer altı su seviyesindeki azalmaların \%60 oranıdan iklimin, geri kalanda ise aşırı su çekilmesinin etkili olduğunu bildirmiştir. Özellikle Konya Kapalı Havzası son 30-40 yıldır yağış olarak kurak bir dönem yaşamaktadır (Doğdu ve ark. 2007; Göçmez ve ark. 2008; Anonim 2015). Bu kuraklık yer altı su seviyelerindeki azalmaları doğrudan etkilemektedir. Konya Karapınar ilçesinde 1980 yıllarından itibaren havzada ölçüm yapılan kuyularda su seviyesindeki düşmenin $0.7 \mathrm{~m} / \mathrm{yll}$ olduğu belirlenmiştir (Doğdu ve ark. 2007).
$\mathrm{Bu} \quad \mathrm{su}$ seviyesindeki değişimlerin pompa performansına etkisinin incelenmesi gerekmektedir.

$\mathrm{Bu}$ çalışmada farklı dalma derinliklerinin milli pompalarin pompaj parametrelerine etkilerinin incelenmesi amaçlanmıştır. Bu bağlamda faklı anma çapına sahip üç adet milli pompanın her birinin üç farklı su giriş ağızında ve farklı dalma derinliği sevilerinde: pompaların toplam dinamik yükseklikleri (TDY), şebekeden çektikleri güç ve gürültü seviyeleri incelenmiştir.

\section{MATERYAL ve METOT}

\section{Materyal}

Denemeler Selçuk Üniversitesi Ziraat Fakültesi Tarım Makineleri ve Teknolojileri Mühendisliği Bölümünde Derin Kuyu Test Ünitesinde yapılmıştır. Test ünitesinin genel ölçüleri ve bazı derin kuyu terminoloji ifadeleri Şekil 1'de verilmiştir.

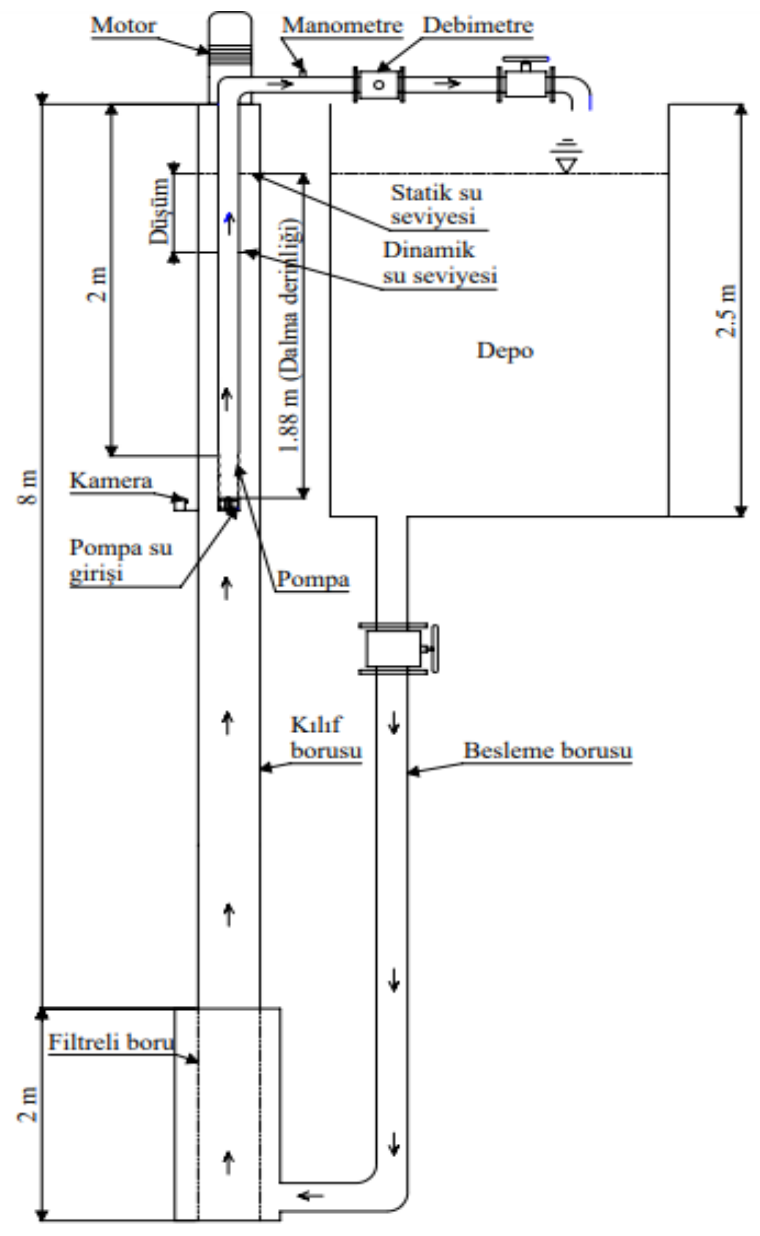

Şekil 1. Derin kuyu test ünitesi Figure 1. Deep well testing unit 
Derin kuyu ile depo arasına çapı 4" ve 6" olan iki adet boru bağlanarak kuyunun beslenmesi sağlanmıştır.

Deneylerde kullanılan düşey tip derin kuyu pompalarının pompa grubu Şekil 2'de ve pompalara ait bazı teknik özellikler ise Çizelge 1'de verilmiştir.

Deneylerde milli pompalarin tahrik edilmesinde kullanılan dik milli elektrik motorunun (Siemens) gücü, çektiği akım değeri ve devir sayısı sırasıyla 5.5 $\mathrm{kW}, 11.1$ A ve 2910 min $^{-1}$ 'dır.

Tüm milli pompaların deneme kombinasyonlarında $2000 \mathrm{~mm}$ uzunluğunda bir adet kolon borusu kullanılmıştır. Çalışmada kullanılan ölçüm aletlerinin bazı teknik özellikleri Çizelge 2'de verilmiştir.

Çizelge 1. Milli pompaların teknik özellik ve ölçüleri

Table 1. Technical characteristics and dimensions of shaft pumps

\begin{tabular}{|c|c|c|c|}
\hline Pompa tipleri & $3 "$ & $4 "$ & $5 "$ \\
\hline Pompa anma çapı (mm) (Pump nominal diameter) & 78 & 105 & 128 \\
\hline Pompa gövde malzemesi (Material of pump body) & Pik & Pik & Pik \\
\hline Pompa çark malzemesi (Material of pump impeller) & Pirinç & Pirinç & Pirinç \\
\hline Pompa mil malzemesi (Material of pump shaft) & $\begin{array}{l}\text { Paslanmaz } \\
\text { çelik }\end{array}$ & $\begin{array}{l}\text { Paslanmaz } \\
\text { çelik }\end{array}$ & $\begin{array}{l}\text { Paslanmaz } \\
\text { çelik }\end{array}$ \\
\hline Giriş kesit alanı $\left(\mathrm{KA}_{2}\right)\left(\mathrm{cm}^{2}\right)$ (Input cross-sectional area) & 72 & 90 & 108 \\
\hline Pompa mili çapı (mm) (Diameter of pump shaft) & 25 & 25 & 25 \\
\hline Pompa kademe sayısı (Number of pump stage) & 2 & 1 & 1 \\
\hline Klerens (mm) (Clearance) & 4.5 & 4.5 & 4.5 \\
\hline Kanat sayısı (adet) (Blade number) & 5 & 5 & 5 \\
\hline Kanat kalınlığı (mm) (Blade thickness) & 5 & 5 & 5 \\
\hline Çark çıkış çapı (mm) (Impeller outlet diameter) & 93.5 & 136 & 150 \\
\hline Çark çıkış genişliği (mm) (Impeller output width) & 15 & 16 & 17.5 \\
\hline
\end{tabular}

Çizelge 2 Kullanılan ölçme aletlerinin bazı teknik özellikleri

Table 2. Some technical features of measuring instruments used

\begin{tabular}{l|l}
\hline Ekipman cinsi & Bazı teknik özellikleri \\
\hline Debimetre (Flowmeter) & $\begin{array}{l}\text { S MAG 100 TİP, DN 80-100-125 flanş bağlantılı elektromanyetik debimetre, 220 V } \\
\text { beslemeli dijital göstergeli, anlık debi, yüzde akış ve toplam gösterimli. Ayarlanabilir } \\
\text { 4-20 m/A plus ve frekans çıkışlı. Ölçüm hatası \%0.5 }\end{array}$ \\
\hline $\begin{array}{l}\text { Manometre } \\
\text { (Manometer) }\end{array}$ & WIKA, 0-10 bar, Alttan Bağlantılı, 4-20 m/A çıkışlı. \\
\hline $\begin{array}{l}\text { Seviye ölçer (Level } \\
\text { meter) }\end{array}$ & $\begin{array}{l}\text { Hydrotechnik marka, 010 tip/1.5 V, } 150 \text { m'lik ölçeklendirilmiş kablolu, ses ve ışık } \\
\text { ikazlı tip. }\end{array}$ \\
\hline $\begin{array}{l}\text { Gürültü ölçer (Noise } \\
\text { meter) }\end{array}$ & $\begin{array}{l}\text { CT-2012 model, giriş } 4 \mathrm{~mA}, \text { güç kaynağı çıkışı DC 24V, Transmitter modeli : TR- } \\
\text { SLT1A4, Ölçüm aralığı:30-80 dBA, 50-100 dB, 80-130 dB, çıkış 4-20 mA, 90-260 ACV } \\
50 H z / 60 H z . ~\end{array}$ \\
\hline $\begin{array}{l}\text { Sicaklık (Temperature } \\
\text { sensors)sensörleri }\end{array}$ & Turck marka, 10-24 VDC, -50...100 0 C, 4-20mA output. \\
\hline Bilgisayar (Computer) & Asus intel core i7. \\
\hline
\end{tabular}

\section{Yöntem}

Denemelerde debi, pozitif basınç, şebekeden çekilen güç, gürültü ve sıcaklık gibi fiziksel büyüklüklerin kaydedilmesi için yazılım ve otomasyon sistemi kullanılmıştır. Sensörlerden alınan bilgiler merkezi bir veri toplama kartı üzerinden kablosuz (Bluetooth) olarak Bilgisayar'a aktarılmaktadır. Merkezi işlemcide depolanan bilgiler bilgisayarda hazırlanan yazılım ara yüzü aracılığı ile operatör tarafından istenilen aralıklarda uygun isimlerle kayıt edilmektedir. Kayıt etme işlemi, saniyede birer adet verileri alabilecek tarzda hazırlanmıştır. Pompa rejime girdikten sonra kayıt işlemine başlanılmış ve bir sensör den 50 adet veri alınmıştır.
Pompaların optimum çalışma devrinde ve her bir pompanın 5 değişik debi aralığında ve 5-7 farklı dalma derinliği seviyesinde ölçümler alınmıştır. Pompa belirlenen herhangi bir debi değerinde çalıştırılarak ilk değerler kayıt altına alındıktan sonra kuyu besleme borularında bulunan vanaların (Şekil 1) kısılması ile dalma derinliği seviyesi değiştirilmiştir. Dalma derinliğinin değişmesi ile değişen debi değeri çıkış borusundaki vana ile tekrar eski haline getirilerek dalma derinliği seviyesi için kayıt işlemi yapılmıştır. Bu şekilde bir debi değeri için 5-7 farklı dalma derinliğinde değerler alınmıştır. Çalışmaya ait deneme planı Şekil 2'de verilmiştir. 


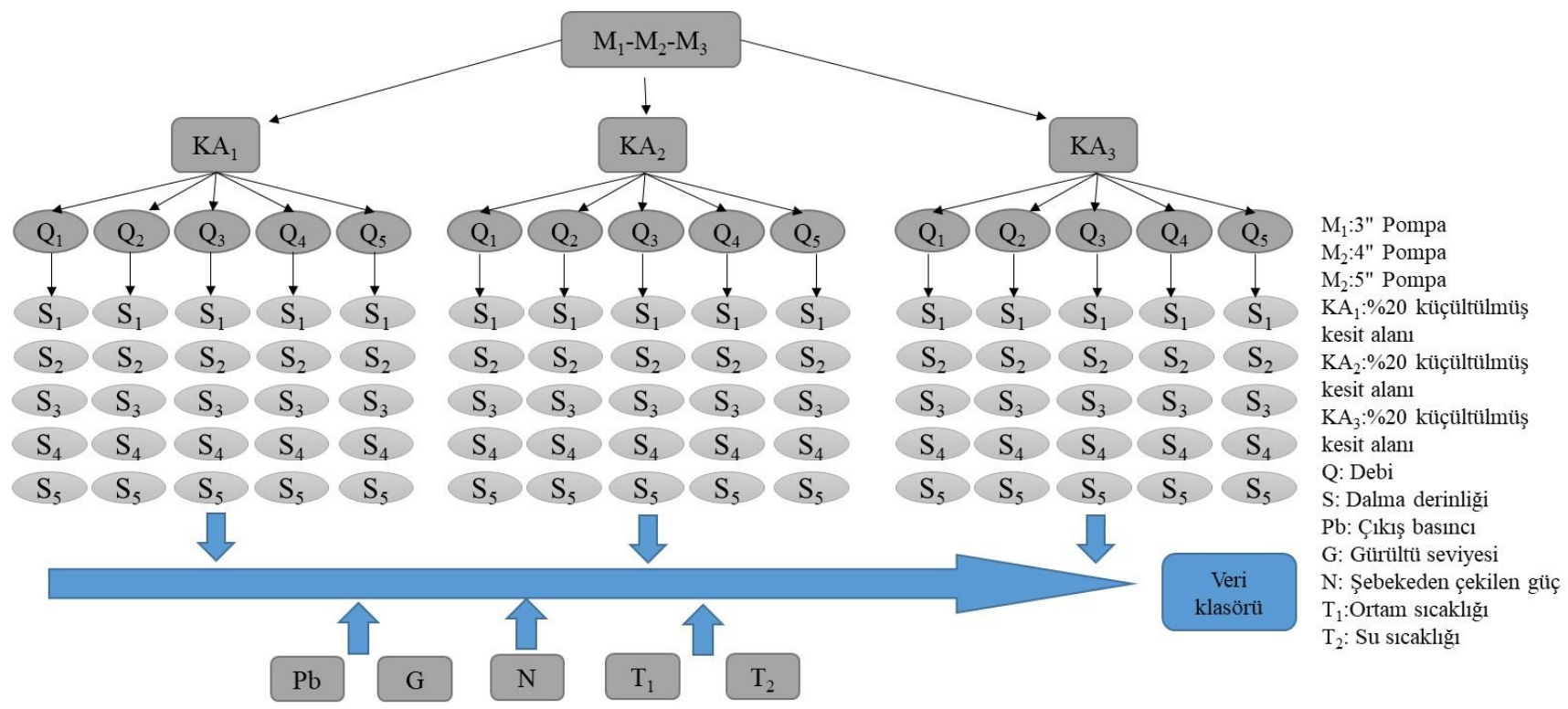

Şekil 2. Deneme deseni

Figure 2. Test design

Pompa 1880 mm sabit hidrolik yük altında denemelere başlanmıştır. Pompa işletme karakteristiklerinin ölçülmesinde ve yapılan hesaplamalarda ilgili standartlar ve literatür dikkate alınmıştır (Tezer 1978; Baysal 1979; Atmaca 1998; Karassik ve ark. 2001; Anonim 2002; Çalışır 2009) Gürültü seviyesi, gürültü ölçme cihazı ile kule kumanda merkezi platformundan pompa kolonu ile kapalı teçhiz borusu arasındaki boşluktan ölçülmüştür (Čdina 2003; Čudina ve Prezelj 2009; Maxime ve Chen Li 2016). Gürültü ölçüm cihazı teçhiz borusu içerisine yerleştirildiği için dış çevre ortamında oluşacak seslerden izole edilmiştir.

Pompaların orijinal giriş ağzı kesit alanına ek olarak, kesit alanını büyütmek için bir adet daha alt adaptör kullanılmıştır. Bu adaptöre geçirilen burç yardımıyla küçük kesit elde edilmiştir (Şekil 3). Çizelge 3'de kesit alanlarının (KA) büyüklükleri verilmiştir.

Pompaların farklı kesit alanlarına, pompanın alt adaptörlerine, kesit şekli bozulmaksızın müdahale edilerek hazırlanmıştır. Pompaların, farklı her bir anma çapı için birer adet (toplam 6 adet) alt adaptör temin edilmiştir. Bunlardan birer tanesi her bir pompa için orijinal kesit alanına sahip $\left(\mathrm{KA}_{2}\right)$ alt adaptör olarak kullanılmıştır. Diğer her bir alt adaptör, pompa anma çapı için orijinal kesit alanına göre yaklaşık \%20 daha büyük kesit alanı oluşturacak şekilde genişletilerek $\mathrm{KA}_{3}$ ölçülerinde elde edilmiştir.

Denemeler süresince ortam sicaklığı ortalaması $18{ }^{\circ} \mathrm{C}$ ve suyun ortalama sicaklığı ise $14{ }^{0} \mathrm{C}$ olarak ölçülmüştür. Pompalar çalıştırılmadan önce $1880 \mathrm{~mm}$ sabit hidrolik yük ve $890 \mathrm{~mm}$ statik su seviyesi yüksekliklerine ayarlamıştır.

Araştırmada pompaların farklı anma çapları, su giriş kesit alanları (KA) ve dalma derinliklerinde (S) toplam dinamik yükseklik (TDY), kuyu içindeki gürültü seviyesi (G), şebekeden çekilen güç (N) değerleri incelenmiştir.

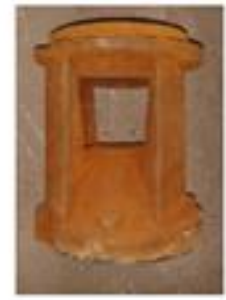

a

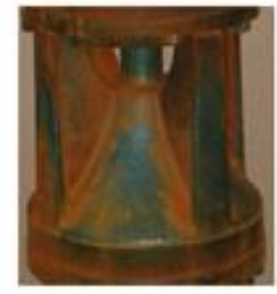

b

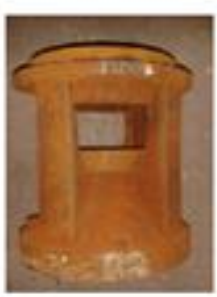

$c$

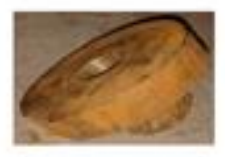

d

Şekil $3 \mathrm{M}_{1}$ pompasına ait farklı giriş ağzı kesit alanları (a: \%20 büyütülmüş, b: orjinal, c: \%20 küçültülmüş) ve değiştirme burcu (d)

Figure 4. Different inlet cross-sectional areas (a: 20\% magnified, b: original, c: 20\% reduced) and replacement device of the $M 1$ pump 
Çizelge 3 Pompalar ve pompa giriş kesit alanları $\left(\mathrm{mm}^{2}\right)$

Table 3. Pumps and pump inlet cross-sectional areas

\begin{tabular}{llll}
\multicolumn{4}{c}{$\left(\mathrm{mm}^{2}\right)$} \\
$\begin{array}{l}\text { Pompalar } \\
\text { (Pumps) }\end{array}$ & $\begin{array}{l}\mathrm{KA}_{1} \\
(\% 20 \\
\text { küçük) } \\
\text { (\%20 Small) }\end{array}$ & $\begin{array}{l}\mathrm{KA}_{2} \\
\text { (Orjinal) } \\
\text { (Orginal) }\end{array}$ & $\begin{array}{l}\mathrm{KA}_{3} \mathbf{( \% 2 0} \\
\text { büyük) } \\
(\% 20 \text { large) }\end{array}$ \\
\hline $\mathrm{M}_{1}$ & 3800 & 4800 & 5800 \\
$\mathrm{M}_{2}$ & 7200 & 9000 & 10800 \\
$\mathrm{M}_{3}$ & 13500 & 16800 & 20000 \\
\hline
\end{tabular}

\section{BULGULAR ve TARTIŞMA}

Pompaların tüm debi değerlerinde ve kesit alanlarında dalma derinliğine bağlı olarak: gürültü seviyesinin ve şebekeden çekilen güç değerlerinin saçılım grafiği incelenmiştir (Şekil 4).

Pompalara ait genel değerlendirme açısından pompa anma çapı arttıkça şebekeden çekilen güç değerleri de genel olarak artmıştır (Şekil 4). $\mathrm{M}_{1}, \mathrm{M}_{2}$ ve $\mathrm{M}_{3}$ pompalarının su giriş kesit alanları ve debilerinde şebekeden çektikleri güç ortalamaları sırasıyla 5.065.20-7.21 kW olarak ölçülmüştür. Pompaların dalma derinliğinin belirli bir seviyeye kadar azalması şebekeden çekilen güç değerlerini genel olarak artırırken dalma derinliklerinin daha da azalması ile şebekeden çekilen güç değerlerinde ani azalmalar saptanmıştır.

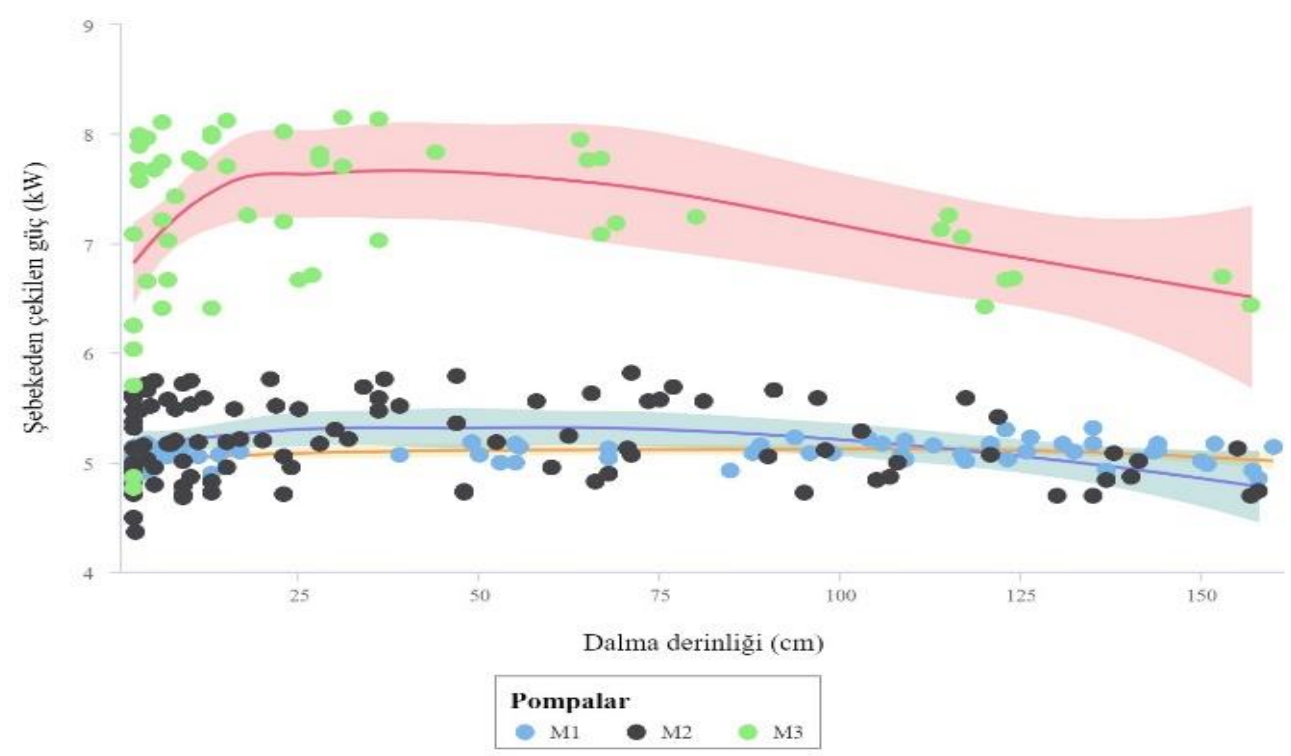

Şekil 4. Pompaların dalma derinliği ile şebekeden çekilen güç değerleri arasıdaki ilişkinin saçılım grafiği Figure 4. Scatter plot belonging to values of submergence and power drawn from the mains the pumps.

Pompaların dalma derinliğine ve gürültü seviyesine ait saçılım grafiği Şekil 5'de verilmiştir. $\mathrm{M}_{1}, \mathrm{M}_{2}$ ve $\mathrm{M}_{3}$ pompalarının su giriş kesit alanları ve debilerinde gürültü seviyeleri ortalamaları sirasıyla 78.58, 80.06 ve $81.2 \mathrm{dBA}$ olarak ölçülmüştür. Pompa anma çapı arttıkça ortalama gürültü seviyesi değeri de artmıştır. Şekil 5 'de görüldüğü gibi pompalarda gürültü seviyesi dalma derinliğine bağlı olarak çok değişim göstermemiştir. Ancak çok düşük dalma derinliklerinde pompalara hava girmesi gürültü seviyesini yükseltmiştir.

Dalma derinliği değişiminin toplam dinamik yükseklik (TDY) üzerine etkisi

Pompa çıkış basıncı $(\mathrm{Pb})$ değerleri, toplam dinamik yüksekliğin hesaplanmasında kullanılan önemli bir parametredir. $\mathrm{Bu}$ nedenle milli pompaların deneme kombinasyonlarında ölçülen $\mathrm{Pb}$ değerleri yardımıyla, toplam dinamik yükseklik (TDY) değerleri hesaplanmış olup değişimleri Şekil 6'da verilmiştir. Denenen milli pompaların sabit debi değerlerinde farklı dalma derinliği seviyelerinde oluşan TDY değerlerinin ortalaması en yüksek $201.6 \mathrm{kPa}$ ile $\mathrm{M}_{3} \mathrm{KA}_{2}$ kombinasyonunda $40 \mathrm{~m}^{3} \mathrm{~h}^{-1}$ lik debi değerinde elde edilirken, en düşük TDY değeri ortalaması ise 86 $\mathrm{kPa}$ değer ile M2KA2 kombihasyonunda $80 \mathrm{~m}^{3} \mathrm{~h}^{-1} \mathrm{lik}$ debi değerinde elde edilmiştir. Her üç milli pompa anma çapı ve giriş kesit alanı seviyelerinde debinin artması ile TDY değerleri azalmıştır.

Dalma derinliğinin toplam dinamik yükseklik (TDY) üzerine etkisi genel olarak değerlendirildiğinde, pompa çıkış basınc değerinin $(\mathrm{Pb})$ aynı debi değerinde düşük dalma derinliklerinde ani olarak düşmesine bağlı TDY değerlerinde de ani düşüşler gözlenmiştir. TDY değerlerindeki ani düşüşler sonucunda pompa verimi olumsuz etkilenmektedir. Yapilan çalışmalarda, yetersiz dalma derinliklerinde vorteks oluşma riskinin bulunduğunu ve emme hattına hava 
girişinin olabileceğini, böylece pompa veriminde azalmalar görülebileceğini Yildirim ve Kocabaş (1998),
Hanson (2000), Karassik ve ark. (2001) araştırmalarında bildirmişlerdir.

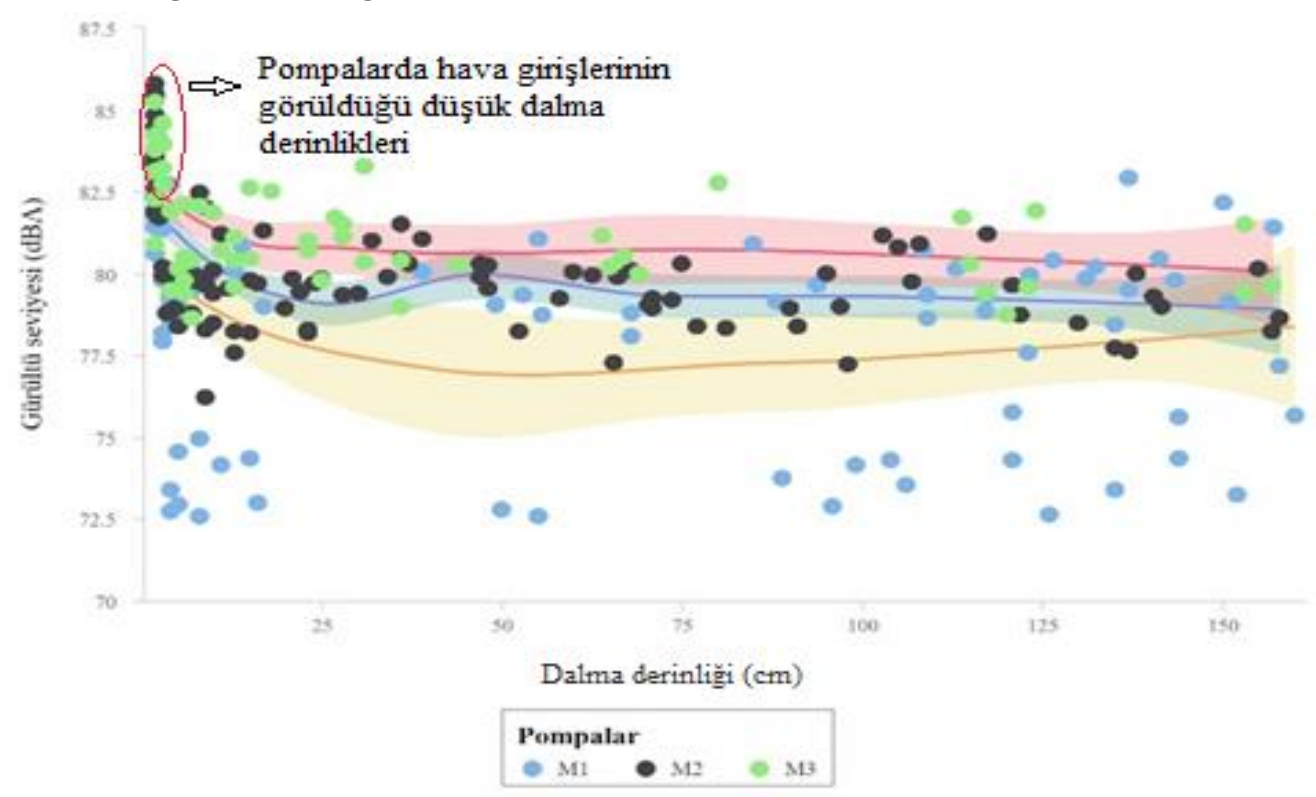

Şekil 5. Pompaların dalma derinliği ile gürültü seviyesi arasındaki ilişkinin saçılım grafiği

Figure 5. Scatter plot belonging to values of submergence and noise level the pumps.
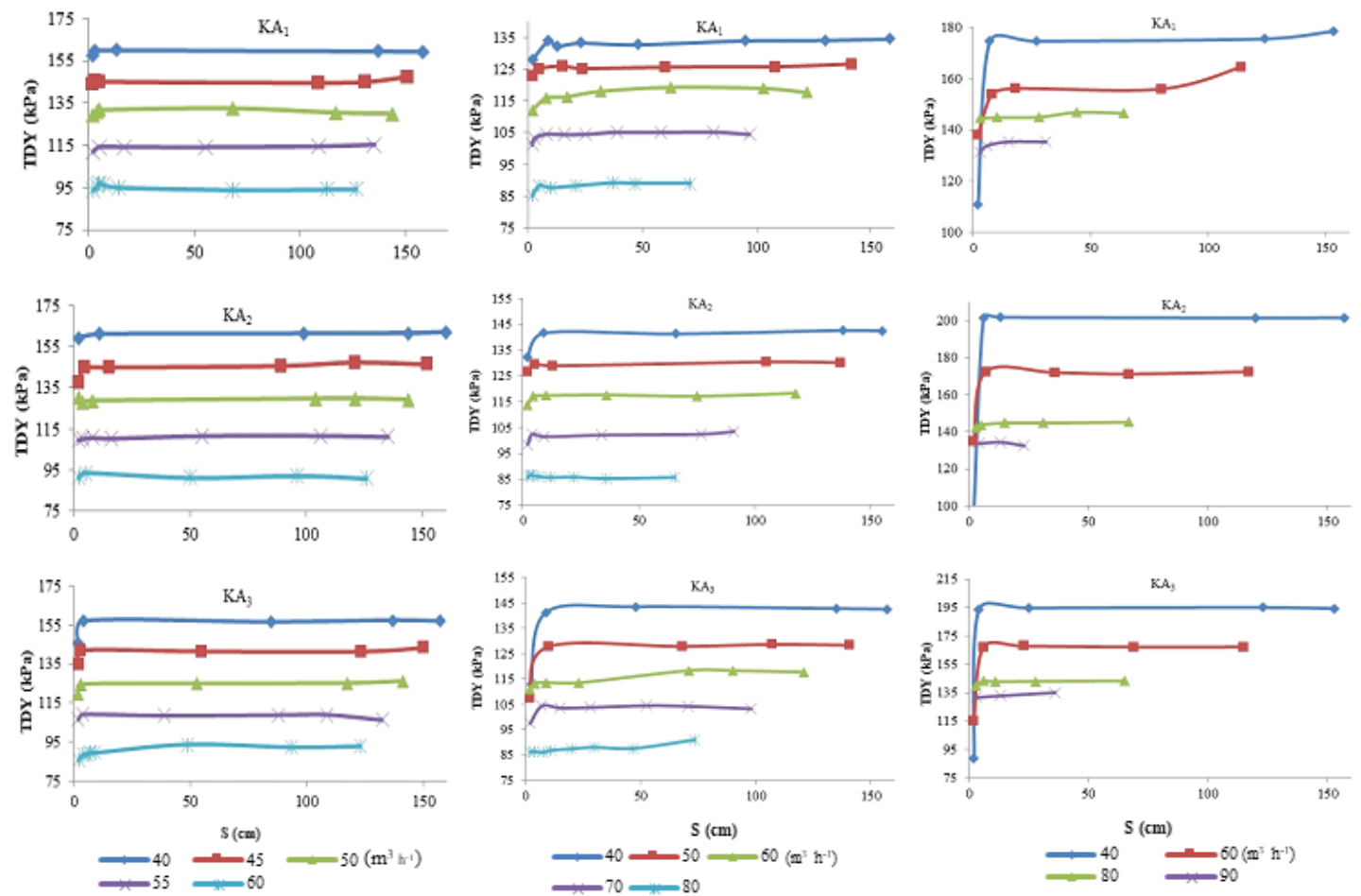

a)

$\mathrm{M}_{1}$

b) $\mathrm{M}_{2}$

c) $\mathrm{M}_{3}$

Şekil 6. Pompaların ve kesit alanlarının dalma derinliğine bağlı toplam dinamik yük (TDY) değişimi Figure 6. Total dynamic load (TDY) change depending on the submergence of the pumps

Dalma derinliğinin çekilen güç üzerine etkisi

Denemeye alınan milli pompaların sabit debi değerlerinde farklı dalma derinliği seviyelerinde çekilen güç değerlerinin ortalamaları incelendiğinde, en yüksek değer $8.09 \mathrm{~kW}$ ile M3KA 1 kombinasyonunda $90 \mathrm{~m}^{3} \mathrm{~h}^{-1}$ debi değerinde elde edilirken, en düşük çekilen güç değeri ise $4.65 \mathrm{~kW}$ ile $\mathrm{M}_{2} \mathrm{KA}_{3}$ kombinasyounda $40 \mathrm{~m}^{3} \mathrm{~h}^{-1}$ debi değerinde elde edilmiştir. $\mathrm{M}_{3}$ pompasının güç tüketim değerinin yüksek çıkması, pompa anma çapının büyümesinden ve debi değerinin yüksek olmasından kaynaklanmaktadır.

M1 pompasının pompa giriş kesit alanına ve dalma derinliğine bağll olarak $60 \mathrm{~m}^{3} \mathrm{~h}^{-1}$ lik debi değerinde 
elde edilen güç değerlerinin değişimi Şekil 7'de, $\mathrm{M}_{2}$ pompası ve $\mathrm{M}_{3}$ pompasına ait değişimler ise aynı debi değerinde Şekil 8 ve 9'da verilmiştir.

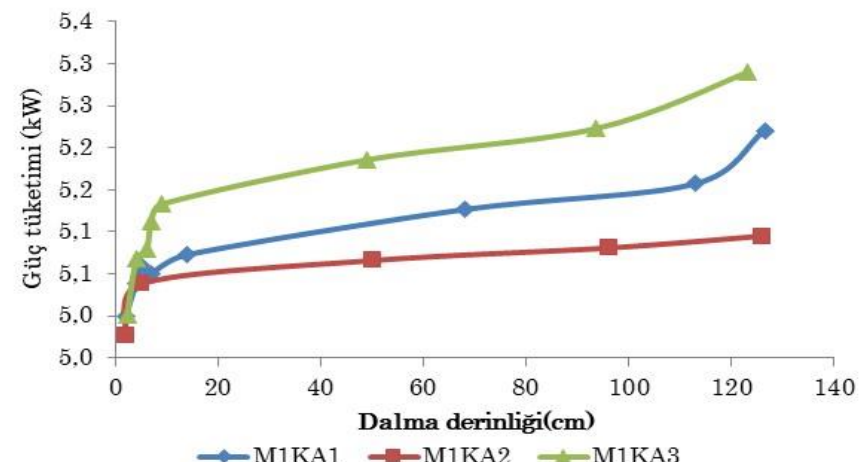

Şekil 7. $\mathrm{M}_{1}$ pompasının $60 \mathrm{~m}^{3} \mathrm{~h}^{-1}$ debi değerinde pompa giriş kesit alanına ve dalma derinliğine bağlı olarak elde edilen güç değerleri

Figure 7. The power values of the $M_{1}$ pump depending on the pump inlet cross-section and the submergence at $60 \mathrm{~m}^{3} \mathrm{~h}^{-1}$ flow rate

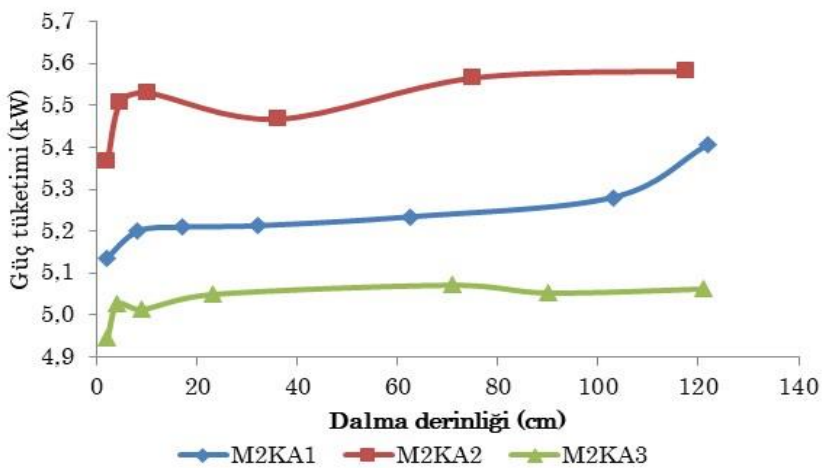

Şekil 8. $\mathrm{M}_{2}$ pompasının $60 \mathrm{~m}^{3} \mathrm{~h}^{-1}$ debi değerinde pompa giriş kesit alanına ve dalma derinliğine bağlı olarak elde edilen güç değerleri

Figure 8. The power values of the $M_{2}$ pump depending on the pump inlet cross-section and the submergence at $60 \mathrm{~m}^{3} \mathrm{~h}^{-1}$ flow rate

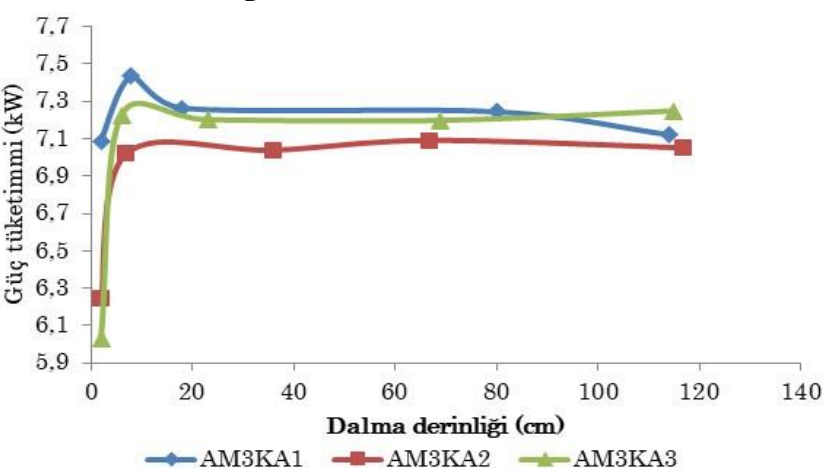

Şekil 9. $\mathrm{M}_{3}$ pompasının $60 \mathrm{~m}^{3} \mathrm{~h}^{-1}$ debi değerinde pompa giriş kesit alanına ve dalma derinliğine bağlı olarak elde edilen güç değerleri

Figure 9. The power values of the $M_{1}$ pump depending on the pump inlet cross-section and the submergence at $60 \mathrm{~m}^{3} \mathrm{~h}^{-1}$ flow rate
Denenen $\mathrm{M}_{2}$ pompasina ait $60 \mathrm{~m}^{3} \mathrm{~h}^{-1}$ lik debi değerinde ve orijinal pompa giriş ağzı alanında $\left(\mathrm{M}_{2} \mathrm{KA}_{2}\right)$ farklı dalma derinliklerinde elde edilen ortalama güç değeri $5.5 \mathrm{~kW}$ olarak saptanmıștır. Kesit alanının değişimine bağlı olarak elde edilen ortalama güç değerleri $\mathrm{M}_{2} \mathrm{KA}_{1}$ 'de $5.26 \mathrm{~kW}$ ve $\mathrm{M}_{2} \mathrm{KA}{ }_{3}$ 'de ise $5.05 \mathrm{~kW}$ olarak bulunmuştur. Şekil 8'de görüldüğü gibi orijinal kesit alanındaki güç değerleri, küçük $\left(\mathrm{M}_{2} \mathrm{KA}_{1}\right)$ ve büyük pompa giriş kesit alanını değerlerinden $\left(\mathrm{M}_{2} \mathrm{KA}_{3}\right)$ daha yüksek bulunmuştur.

Şekil 7'nin incelenmesiyle $60 \mathrm{~m}^{3} \mathrm{~h}^{-1}$ sabit debi değerinde $\mathrm{M}_{1}$ milli pompasının orijinal kesit alanı olan $\mathrm{M}_{1} \mathrm{KA}_{2}$ 'de farklı dalma derinliklerinde elde edilen güç değerlerinin ortalaması $5.07 \mathrm{~kW}$ olarak tespit edilmiştir. Kesit alanının değişimine bağlı olarak elde edilen güç değerlerinin ortalaması $\mathrm{M}_{1} \mathrm{KA}_{1}$ 'de $5.1 \mathrm{~kW}$ ve $\mathrm{M}_{1} \mathrm{KA}_{3}$ 'de ise $5.16 \mathrm{~kW}$ olarak bulunmuştur. Orijinal kesit alanı değerlerinden daha küçük kesit $\left(\mathrm{M}_{1} \mathrm{KA}_{1}\right)$ ve daha büyük pompa giriş kesit alanın da $\left(\mathrm{M}_{1} \mathrm{KA}_{3}\right)$ güç değerleri biraz daha düşük değerlerde bulunmuştur.

$\mathrm{M}_{3}$ milli pompasının $60 \mathrm{~m}^{3} \mathrm{~h}^{-1} \mathrm{lik}$ debi değerinde $\mathrm{M}_{3} \mathrm{KA}_{2}$ 'de kombinasyonunda farkl dalma derinliklerinde belirlenen ortalama güç değerleri 7.05 $\mathrm{kW}$ olarak bulunmuştur. Kesit alanının değişimine bağlı olarak elde edilen ortalama güç değerleri küçük pompa giriş kesit alanında $\left(\mathrm{M}_{3} \mathrm{KA}_{1}\right) 7.27 \mathrm{~kW}$ ve büyük giriş kesit alanında $\left(\mathrm{M}_{3} \mathrm{KA}_{3}\right)$ ise $7.2 \mathrm{~kW}$ olarak bulunmuştur. Küçük giriş kesit alanında ve büyük giriş kesit alanında elde edilen güç değerleri, orijinal giriş kesit alanı değerlerinden daha yüksek bulunmuştur. Pompaların güç tüketimi bakımından $\mathrm{M}_{1}$ ve $\mathrm{M}_{3}$ pompasinda $\mathrm{KA}_{2}$ kesit alanının, $\mathrm{M}_{2}$ pompasında ise $\mathrm{KA}_{3}$ kesit alanının kullanılması uygun olacaktır.

Şekil 7, 8 ve 9'da görüleceği üzere milli pompaların düşük dalma derinliği değerlerindeki güç tüketiminde ani düşüşler meydana gelmiştir. Şebekeden çekilen gücün ani düşmeye başladığı noktaya karşılık gelen dalma derinliği değeri, kritik dalma derinliğinin aşılmaya başlandığını göstermektedir. Diğer bir deyişle vorteksin oluşmaya başladığı derinlik olarak değerlendirilebileceği söylenebilir.

\section{Dalma derinliğinin gürültü seviyesine etkisi}

Denemeye alınan milli pompaların sabit debi değerlerinde farklı dalma derinliği seviyelerinde oluşan gürültü düzeylerinin ortalamaları en yüksek 83.13 dBA ile $\mathrm{M}_{3} \mathrm{KA}_{1}$ kombinasyonunda $90 \mathrm{~m}^{3} \mathrm{~h}^{-1}$ lik debi değerinde elde edilirken, en düşük gürültü seviyesi ortalaması ise $74.47 \mathrm{dBA}$ değer ile $\mathrm{M}_{1} \mathrm{KA}_{2}$ kombinasyonunda $55 \mathrm{~m}^{3} \mathrm{~h}^{-1}$ lik debi değerinde elde edilmiştir. Pompaların tüm debi ve kombinasyonlarında, pompanın suyu emmesi esnasında, özellikle düşük dalma derinliklerinde hava girişi ile gürültü seviyesi değerleri ani olarak yükselmiştir. $\mathrm{M}_{3}$ pompasında gürültü seviyesinin yüksek çıkması anma çapının büyük olmasına ve debi 
değerinin yüksek olmasına bağlanabilir. $\mathrm{M}_{1}$ pompasında ise gürültü değerlerinin düşük çıkması, pompa anma çapının küçük olmasından ve pompanın optimum çalışma noktasına yakın debi değerlerinde çalışmasından kaynaklanmıştır. Çalışır ve ark. (2006), araştırmasında, farklı yatay milli santrifüj pompaların optimum debi değerlerinde en düşük gürültü değerlerinin elde edildiğini bildirmektedirler.

$\mathrm{M}_{1}$ pompasının pompa giriş kesit alanına ve dalma derinliğine bağlı olarak $60 \mathrm{~m}^{3} \mathrm{~h}^{-1}$ lik debi değerinde elde edilen gürültü seviyelerinin değişimi Şekil 10'da, $\mathrm{M}_{2}$ pompası ve $\mathrm{M}_{3}$ pompasına ait değişimler ise aynı debi değerinde Şekil 11 ve 12'de verilmiştir.

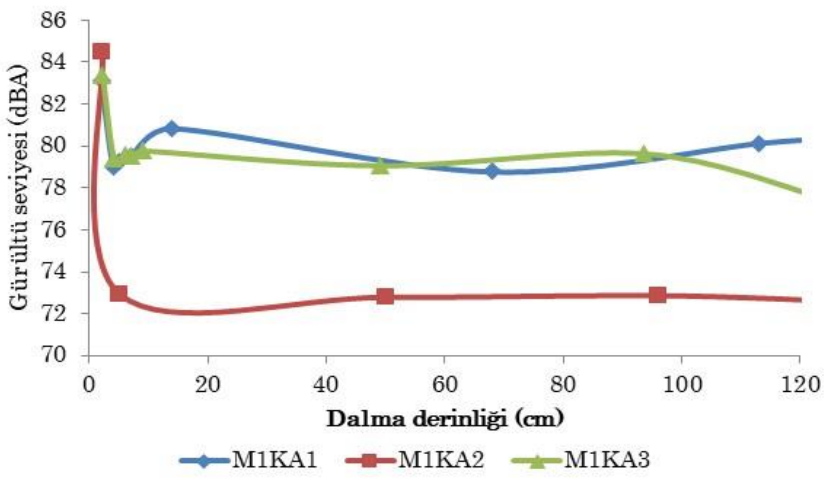

Şekil 10. $\mathrm{M}_{1}$ pompasının $60 \mathrm{~m}^{3} \mathrm{~h}^{-1}$ debi değerinde pompa giriş kesit alanına ve dalma derinliğine bağlı olarak elde edilen gürültü değerleri

Figure 10. The noise values of the $M_{1}$ pump depending on the pump inlet cross-section and the submergence at $60 \mathrm{~m}^{3} \mathrm{~h}^{-1}$ flow rate

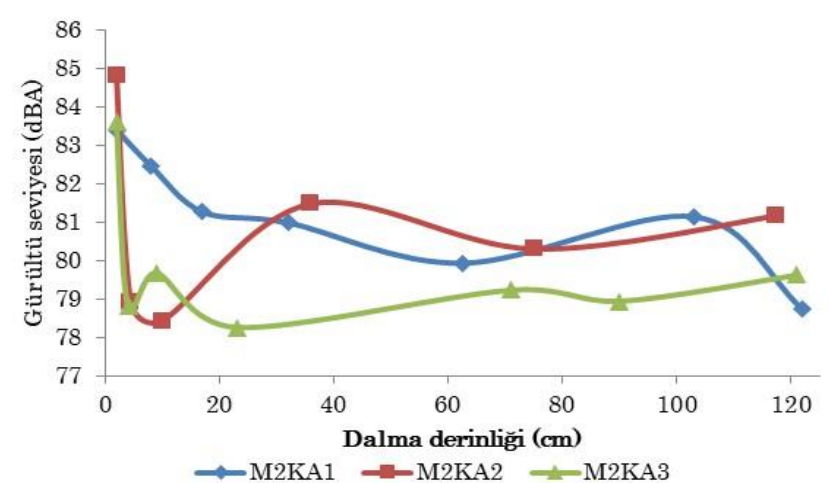

Şekil 11. $\mathrm{M}_{2}$ pompasının $60 \mathrm{~m}^{3} \mathrm{~h}^{-1}$ debi değerinde pompa giriş kesit alanına ve dalma derinliğine bağlı olarak elde edilen gürültü değerleri

Figure 11. The noise values of the $M_{2}$ pump depending on the pump inlet cross-section and the submergence at $60 \mathrm{~m}^{3} \mathrm{~h}^{-1}$ flow rate

Şekil 10'un incelenmesiyle $60 \mathrm{~m}^{3} \mathrm{~h}^{-1}$ sabit debi değerinde $\mathrm{M}_{1}$ milli pompasının orijinal kesit alanı olan $\mathrm{M}_{1} \mathrm{KA}_{2}$ 'de farklı dalma derinliklerinde elde edilen ortalama gürültü değerleri $72.90 \mathrm{dBA}$ olarak tespit edilmiştir. Kesit alanının değişimine bağlı olarak elde edilen ortalama gürültü değerleri $\mathrm{M}_{1} \mathrm{KA}_{1}$ 'da $79.70 \mathrm{dBA}$ ve $\mathrm{M}_{1} \mathrm{KA}{ }_{3}$ 'de ise $79.20 \mathrm{dBA}$ olarak bulunmuştur. Orijinal kesit alanı değerlerinden daha küçük kesit alanında $\left(\mathrm{M}_{1} \mathrm{KA}_{1}\right)$ ve büyük kesit alanının da $\left(\mathrm{M}_{1} \mathrm{KA}_{3}\right)$ gürültü değerleri daha yüksek bulunmuştur.

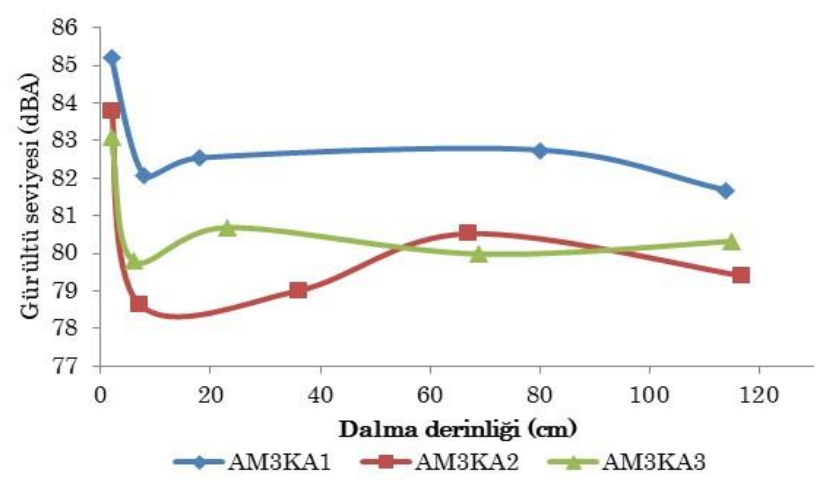

Şekil 12. $\mathrm{M}_{3}$ pompasının $60 \mathrm{~m}^{3} \mathrm{~h}^{-1}$ debi değerinde pompa giriş kesit alanına ve dalma derinliğine bağlı olarak elde edilen gürültü değerleri

Figure 12. The noise values of the $M_{3}$ pump depending on the pump inlet cross-section and the submergence at $60 \mathrm{~m}^{3} \mathrm{~h}^{-1}$ flow rate

Denemede kullanılan $\mathrm{M}_{2}$ pompasina ait $60 \mathrm{~m}^{3} \mathrm{~h}^{-1}$ lik debi değerinde ve orijinal pompa giriş ağzı alanında $\left(\mathrm{M}_{2} \mathrm{KA}_{2}\right)$ farkl 1 dalma derinliklerinde elde edilen ortalama gürültü değeri 80.87 dBA olarak saptanmıştır. Kesit alanının değişimine bağlı olarak elde edilen ortalama gürültü değerleri $\mathrm{M}_{2} \mathrm{KA}_{1} 80.7$ dBA ve $\mathrm{M}_{2} \mathrm{KA}_{3}$ ise $79.10 \mathrm{dBA}$ olarak bulunmuştur. Şekil 11'de görüldüğü gibi pompanın farklı giriş ağzı kesit alanlarında elde edilen gürültü değerlerinin farklı olmadığı görülmektedir.

$\mathrm{M}_{3}$ milli pompasının $60 \mathrm{~m}^{3} \mathrm{~h}^{-1}$ lik debi değerinde $\mathrm{M}_{3} \mathrm{KA}_{2}$ kombinasyonunda farklı dalma derinliklerinde belirlenen ortalama gürültü seviyesi değerleri 79.39 dBA olarak bulunmuştur. Kesit alanının değişimine bağlı olarak elde edilen ortalama gürültü değerleri küçük giriş kesit alanında $\left(\mathrm{M}_{3} \mathrm{KA}_{1}\right) 82.25 \mathrm{dBA}$ ve büyük giriş kesit alanında $\left(\mathrm{M}_{3} \mathrm{KA}_{3}\right)$ ise $80.20 \mathrm{dBA}$ olarak bulunmuştur. Küçük giriş kesit alanında ve büyük giriş kesit alanında elde edilen gürültü değerleri, orijinal kesit alanı değerlerinden daha yüksek bulunmuştur.

Aynı debi değerinin $\left(60 \mathrm{~m}^{3} \mathrm{~h}^{-1}\right)$ farklı dalma derinliklerinde ölçülen gürültü seviyelerinin ortalamaları pompa anma çapı artıkça artış göstermiştir. Pompaların giriş kesit alanlarının değişiminin gürültü seviyesinde etkili olduğu ortaya çıkmıştır.

Genel olarak milli pompalarda değişik dalma derinliklerinde elde edilen ortalama gürültü değerlerinin orijinal kesit alanlarına bağlı olarak yüksek çıkmasına, değiştirilen alt adaptörün pompa çarkı ile olan uyumsuzluğu ve pompaların çalışma 
esnasında dalma derinliklerine bağlı olarak debi değerlerinde görülen dalgalanmaların $\left(\mathrm{M}_{2}\right.$ ve $\mathrm{M}_{3}$ pompalarında optimum debiden uzak debi değerlerinde çalışması) etkili olduğu söylenebilir. Ayrıca Şekil 10, 11 ve 12'nin incelenmesiyle gürültü değerlerinin pik yaptığı dalma derinliklerinin düşük değerlerde olması, pompanın vorteks oluşumuna başladığı anlamına gelmektedir. Diğer bir deyişle dış çevre koşullarından arındırılmış gürültü seviyesi ölçümleri ile vorteks oluşumunun başlama anı ile ilgili dalma derinliklerinin belirlenebileceği söylenebilir. Benzer sonuçları Čdina (2003); Čudina ve Prezelj (2009) yaptıkları çalışmalarında pompaların ses frekanslarinın vorteks (kavitasyon) durumunda, pompanın değişik çalışma rejimlerine göre net bir şekilde ayrıştığını bildirmektedirler.

\section{SONUÇ ve ÖNERILER}

Pompa anma çapı arttıkça şebekeden çekilen güç ve gürültü seviyesi ortalamaları artmıştır. Pompaların dalma derinliğinin belirli bir seviyeye kadar azalması şebekeden çekilen güç değerlerini artırmıştır. Pompaların çok düşük dalma derinliklerinde şebekeden çekilen güç değeri azalırken gürültü seviyeleri artmıştır. Pompaların $\mathrm{KA}_{1}, \mathrm{KA}_{2}$ ve $\mathrm{KA}_{3}$ kesit alanları bakımından şebekeden çektikleri güç ortalamaları sırası ile $5.89,5.84$ ve $5.73 \mathrm{~kW}$ gürültü seviyesi ortalamaların ise sirasiyla $80.69,78.69$ ve 80.47 dBA olarak bulunmuştur. Pompalarm şebekeden çektikleri güç bakımından $\mathrm{KA}_{3}$ su giriş kesitinin, gürültü seviyesi bakımından ise $\mathrm{KA}_{2}$ su giriş kesitinin kullanılması uygundur.

Düşük dalma derinliklerinde pompa girişlerinde vorteks oluşması ve hava girmesi şebekeden çekilen güç değerlerinde ani düşümlere sebep olurken gürültü seviyelerinde ani yükselmelere neden olmuştur. Özellikle arazi şartlarında seviye ölçümlerinin zor olduğu derin kuyu pompalarında düzenli debi, güç ve gürültü ölçümleri ile pompaların statik ve dinamik seviye durumu, çalışma karakteristiğinin değişimi rahatlıkla takip edilebilir.

\section{TEŞEKKÜR}

$\mathrm{Bu}$ çalışma, Türkiye Bilimsel ve Teknik Araştırma Kurumu (TÜBITAK, Proje No: 213O140) tarafindan desteklenmiştir. $\mathrm{Bu}$ çalışmaya katkıda bulunan merhum Prof.Dr. Sedat ÇALIŞIR Hocamıza teşekkür ederiz.

\section{Araştırmacıların Katkı Oranı Beyan Özeti}

Yazarlar makaleye eşit oranda katkı sağlamış olduklarını beyan eder.

\section{Çıkar Çatışması Beyanı}

Makale yazarları aralarında herhangi bir çıkar çatışması olmadığını beyan ederler.

\section{KAYNAKLAR}

Anonim 2002. Rotodinamik Pompalar-Hidrolik Performans Kabul Deneyleri Sinif 1 ve Sinif 2 (TS EN ISO 9906). Türk Standardları Enstitüsü, Ankara.

Anonim 2015. Konya Havzası Kuraklık Yönetim Planı. T.C. Orman ve Su İşleri Bakanlığ $\mathrm{Su}$ Yönetimi Genel Müdürlüğü Taşkın ve Kuraklık Yönetimi Dairesi Başkanlığı, Ankara.

Anonim 2016. Devlet Su İşleri Genel Müdürlüğü 2016 Yll Faliyet Raporu. http://strateji.dsi.gov.tr/ docs/belgeler/dsi-2016-faaliyet-raporu.pdf?sfvrsn=0 (Alınma Tarihi :18.02.2020)

Atmaca S 1998. Dalgıç Pompalara Uygulanan Pompa Kabul Deneyleri. 3. Pompa kongresi, 24-26 Eylül 1998, İstanbul.

Baysal K 1979. Tam Santrifüj Pompalar: Hesap, Çizim ve Konstrüksiyon Özellikleri, İstanbul Teknik Üniversitesi, 24 sy.

Čdina M 2003. Detection of Cavitation Phenomenon in a Centrifugal Pump Using Audible Sound. Mechanical Systems And Signal Processing 17 (6): 1335-1347.

Chen Y, Wu C, Wang B, Du M, 2012. ThreeDimensional Numerical Simulation of Vertical Vortex At Hydraulic Intake. Procedia Engineering 28: 55-60.

Čudina M, Prezelj J, 2009. Detection Of Cavitation In Operation Of Kinetic Pumps. Use of Discrete Frequency Tone in Audible Spectra. Applied Acoustics 70 (4): 540-546.

Çalışır S, Aydın C, Mengeş H O, 2006. Derin Kuyu Pompaj Tesislerinde Titreşim Hızı ve Gürültü Düzeyinin Belirlenmesi. Selçuk Tarım Bilimleri Dergisi 20 (38): 49-54.

Çalışır S, 2009. Sulamada Pompaj Tesisleri (Tarım Makineleri Kitabı. Edt:Ergüneş G, Nobel Yayın Dağıtım, Ankara) 351-413.

Doğdu M, Toklu M, Sağnak C, 2007. Konya Kapalı Havzası'nda Yağış ve Yeraltısuyu Seviye Değerlerinin İrdelenmesi. 1. Türkiye İklim Değişikliği Kongresi 11 - 13 Nisan 2007, İstanbul.

Driscoll F 2010. Kuyu Hidroliği. DSİ, Ankara, 88 sy.

Göçmez G, Dıvrak B, Galena İ 2008. Konya Kapalı Havzası'nda Yeraltı Suyu Seviyesinin Değişiminin Tespiti Özet Raporu. Ofset Yapımevi, İstanbul, 82 sy.

Hanson B 2000. Irrigation Pumping Plants (UC Irrigation and Drainage Specialist. Department of Land, Air and Water Resources, University of California, Davis) 69-73.

Karassik IJ, Messina JP, Cooper P, Heald CC 2001. Pump handbook, McGraw-Hill New York, 1824 sy.

Maxime B, Chen Li F 2016. Cavitation Effects in Centrifugal Pumps-A Review. Int. Journal of Engineering Research and Application 6 (5):52-63. 
Nagahara T, Sato T, Okamura T 2001. Effect of the Submerged Vortex Cavitation Occurred in Pump Suction İntake on Hydraulic Forces of Mixed Flow Pump İmpeller. Proc. $4^{\text {th }}$ International Symposium on Cavitation June 20-23 2001, Pasadena, USA.

Tezer E 1978. Sulamada Pompaj Tesisleri (Proje, Seçim ve İşletme Yöntemleri) Cilt 1-2-3. Çukurova Üniversitesi Ziraat Fakültesi Yayınları, Adana.
Yıldırım N, Kocabaş F 1998. Critical Submergence For Intakes in Still-Water Reservoir. Journal of Hydraulic Engineering 124 (1): 103-104.

Yıldırım N, Akay H, Taştan K 2011. Critical Submergence for Multiple Pipe Intakes by Potential Solution (Technical Note). Journal of Hydraulic Research 49 (1): 117-121. 